\title{
Author Correction: X-ray photonics: Attosecond XFEL for pump-probe experiments
}

Heung-Sik Kang $\mathbb{D}$ and In Soo Ko

Correction to: Nature Photonics https://doi.org/10.1038/s41566-019-0570-8, published online 20 December 2019.

We would like to make the readers aware that in this News \& Views article, the following text should have been attributed to ref. 4 (A. Mak et al. Rep. Prog. Phys. 82, 025901; 2019):

"The realm of attosecond science emerged when the attosecond $\left(1\right.$ as $\left.=10^{-18} \mathrm{~s}\right)$ timescale was reached by high-harmonic generation ${ }^{8}$ (HHG). Attosecond pulses have opened the possibilities to image and control the behaviour of electron wave packets within atoms."

"High-intensity attosecond pulses are required to fully explore the new field of attochemistry, where the motion of electrons and their influence on chemical reactions are investigated. APAP methods could be applied to studies of charge migration, which is a fundamental process in biology."

"...the investigation of core-electronic coupling as well as correlation and quantum coherence of valence electrons and holes."

No changes have been made to the above text in the News \& Views article.

In addition, in the sentence beginning "Since the bandwidth of the XFEL...", ref. 8 was incorrectly cited instead of ref. 5. This has now been corrected online.

Published online: 5 March 2020

https://doi.org/10.1038/s41566-020-0610-4

๑) Springer Nature Limited 2020 